\title{
Implantación de un sistema de gestión de seguridad y Salud en el trabajo basado en el modelo Ecuador
}

\section{Implementation of a security management system and Health at work based on the Ecuador model}

\section{Implementação de um sistema de gestão de saúde e segurança no trabalho baseado no modelo do Equador}

Cesar A. Arias-Mendoza I

arias.uleam@hotmail.com

Recibido: 30 de enero de $2017 *$ Corregido: 20 de febrero de $2017 *$ Aceptado: 20 junio de 2017

I. Magister en Seguridad Salud y Ambiente Laboral, Ingeniero Industrial Profesor de la Universidad Laica Eloy Alfaro de Manabí, Manta, Ecuador. 


\section{Resumen}

La normativa legal vigente del Ecuador establece la obligatoriedad de la implementación del sistema de gestión de seguridad y salud en el trabajo en todas las instituciones públicas y privadas del país, indistinto si es una empresa manufacturera o de servicios. En este sentido, el Instituto Ecuatoriano de Seguridad Social (IESS) ha adoptado la metodología denominada "Modelo Ecuador" para el sistema, estableciendo cuatro pilares de gestión: administrativa, técnica, talento humano y procedimientos operativos básicos. Considerando estas premisas, este trabajo tiene como objetivo implementar un sistema de gestión de seguridad y salud basado en el "Modelo Ecuador" para la Unidad Educativa Particular "Isaac Newton" de la cuidad de Manta, considerando la totalidad de sus colaboradores en sus distintas áreas. La metodología aplicable tiene su base en la Resolución 333 del reglamento para el Sistema de Auditoria de Riesgo del Trabajo (SART) del IESS, que permitió desarrollar el diagnóstico de la situación inicial de la institución, fundamentado en un estudio descriptivo, observación directa, y contraste con la normativa legal, arrojando un nivel de cumplimiento del 6,76\%. El análisis de los factores de riesgo en los puestos de trabajos se desarrolló con base a la matriz destinada por el del Ministerio de Relaciones Laborales y la metodología de evaluación general de riesgos del Instituto Nacional de Seguridad e Higiene de España (INSHT). El diseño del sistema de gestión está conformado por la planificación, procedimientos, métodos, formatos y documentos relacionados destinados a su implantación. Este sistema se sustenta en el alcance del Índice de Eficiencia del 68,02\% y en la propuesta de implantación del 23,37\% adicional, considerando satisfactorio, y apoyado en el análisis favorable de la relación beneficio-costo.

Palabras clave: Gestión de seguridad; Modelo Ecuador; Índice de Eficiencia. 


\section{Abstract}

In Ecuador, the current legal regulations require the implantation of safety and health occupational management systems in all public and private institutions, regardless if this is a manufacturing or a service company. Meaning, the Ecuadorian Institute of Social Security (IESS in Spanish) has adopted the methodology known as "The Ecuador Model” (El Modelo Ecuador) to the system, it's a four pillars system: administrative management, technical management, human resource management and basic operating process. With these issues in mind, this thesis has as an objective to implement a safety and health occupational management system based on Ecuador Model at "Isaac Newton" educational institute in the city of Manta, considering its employees in different areas. The applicable methodology is based in the Resolution 333, Regulation of Occupational Risk Audits System (SART in Spanish) from IESS; it enabled us to run the diagnostics of the initial situation of the institution, based in a descriptive study, direct observation in contrast with legal regulations, which showed an accomplishment level of $6,76 \%$. The analysis of risk factors in the workplace was developed according to the matrix from The Ministry of Labor Relations and the general methodology of risk evaluation from The National Institute for Health and Safety in Spain (INSHT in Spanish). The management system design is consisting of the planning, procedures, methods, formats as well as relevant documents for its implementation. This system has an $68,02 \%$ efficiency index, with an 23,37\% additional proposed implantation, which is considered satisfactory, and supported in a favourable cost-benefit analysis.

Key words: Security management; Ecuador Model; Efficiency Index. 


\section{Introducción.}

La gestión de la seguridad y salud en el trabajo, se está consolidando a nivel mundial, como un pilar necesario de toda empresa para mejorar las condiciones de sus trabajadores alcanzando una mayor productividad y por consiguiente, mayor rentabilidad de su actividad laboral. Alrededor del mundo, este beneficio ha llevado que varias instituciones no gubernamentales y de instancia de derecho público, hayan desarrollado metodologías de aplicación para la gestión de la seguridad y salud ocupacional, con clara líneas en común. [1-5]

El Ecuador, considerando los lineamientos mundiales con base en la protección del trabajador, se encuentra en un período de transición y solidificación de sus leyes sobre seguridad y salud en el trabajo; es así, que ratifica desde el año 2005, instrumentos internacionales y adapta normativa interna para garantizar los derechos de los trabajadores reconocidos a nivel externo. [6-8]

Desde el 2008, el Ecuador, con la aprobación de su nueva Constitución, dentro de su capítulo sexto: trabajo y producción, en su sección tercera, artículo 326, establece los principios del derecho al trabajo, y entre ellos, en su numeral 5 indica que: "toda persona tendrá derecho a desarrollar sus labores en un ambiente adecuado y propicio, que garantice su salud, integridad, seguridad, higiene y bienestar"; consagrando como garantía constitucional la seguridad y salud ocupacional (Asamblea Nacional del Ecuador, 2008). [9-12]

Siguiendo con estos lineamientos, en el año 2010, adopta la Resolución 333: Reglamento para el Sistema de Auditoría de Riesgos de Trabajo SART, y el año 2011, el Consejo Ejecutivo del Instituto Ecuatoriano de Seguridad Social adopta la Resolución 390, que estima en su artículo 51, la 
obligatoriedad de la implementación del Sistema de Gestión de Seguridad y Salud en el Trabajo (SGSST) bajo parámetros establecidos por la autoridad pública. [13-18]

Dentro del Plan Nacional del Buen Vivir, articulado por la Secretaria Nacional de Planificación y Desarrollo del Ecuador para el periodo 2013 -2017, se establece la postura política y procesos que el país espera tener en el periodo, considerando como prioritario el establecimiento de políticas públicas referente el tema de seguridad y salud ocupacional. Es por esto, que dentro de su objetivo 3: mejorar la calidad de vida de la población, plantea a la salud con un mirada intersectorial que busca garantizar condiciones de hábitat dignos, promoción de la misma y la prevención de enfermedades que garanticen el adecuado fortalecimiento de las capacidades de las personas y mejorando su calidad de vida. Con el objeto de alcanzar este lineamiento, se plantea su política 3.8 literal e, que busca impulsar la implementación de planes de salud y seguridad ocupacional en las empresas públicas y privadas del país (Secretaría Nacional de Planificación y Desarrollo, 2013). [1419]

Esta política se enriquece de argumentos con base en el objetivo diez del plan anteriormente mencionado, que busca impulsar la transformación de la matriz productiva, convirtiendo el país de mono-exportador agrícola a exportador de bienes o servicios con valor agregado, pretendiendo la industrialización del país, y por consiguiente el establecimiento de sistema de gestión de calidad, seguridad, medio ambiente y responsabilidad social.

Sin embargo, en la Unidad Educativa Particular "Isaac Newton", no se cuenta con un Sistema de Gestión de Seguridad y Salud en el Trabajo regido por la normativa vigente, que permita controlar y minimizar los riesgos a los que se encuentran expuestos sus trabajadores, quienes desarrollan prioritariamente actividades administrativas y pedagógicas. 
El objetivo de la presente investigación es implantar un Sistema de Gestión de Seguridad y Salud en el Trabajo basado en el "Modelo Ecuador" en la Unidad Educativa Particular "Isaac Newton", garantiza el cumplimiento de la normativa legal vigente.

\section{Metodología.}

La presente revisión de literatura busca determinar un Sistema de Gestión de Seguridad y Salud en el trabajo; literatura que fue citada de las siguiente bases de datos científicas:; REDLAYC, SCIELO, SCOPUS.

\section{Revisión de Literatura.}

Sistemas de Gestión de Seguridad y Salud

La gestión de la seguridad y salud en el trabajo y la implantación de su sistema, se han convertido en nuevo tema en el Ecuador, aunque legalmente no lo sean; transformándose, de ser un requisito de obligatorio cumplimiento por las organizaciones, a un modelo de permanencia y eficiencia productiva en las mismas.

Es primordial conocer el significado de un sistema de gestión dentro de una empresa, y para ello es necesario descomponer sus términos: la palabra sistema, según (Real Academia Española, 2014) la define como "el conjunto de elementos que relacionados entre sí ordenadamente contribuyen a determinado objetos", y a la palabra gestión, la define como "la acción o efecto de hacer actividades para el logro de un negocio o un deseo cualquiera". [20-29] 
Los términos seguridad y salud, dentro de este ámbito, toman mayor importancia. Así, el Instrumento Andino de Seguridad y Salud en el Trabajo (Decisión 584), define al conjunto de este sistema como el:

“conjunto de elementos interrelacionados o interactivos que tienen por objeto establecer una política y objetivos de seguridad y salud en el trabajo, y los mecanismos y acciones necesarios para alcanzar dichos objetivos, estando íntimamente relacionado con el concepto de responsabilidad social empresarial, en el orden de crear conciencia sobre el ofrecimiento de buenas condiciones laborales a los trabajadores, mejorando de este modo la calidad de vida de los mismos, así como promoviendo la competitividad de las empresas en el mercado" (Comunidad Andina, 2005). [30-35]

Considerando a la seguridad y salud como derechos fundamentales y desechando su concepto restringido de sólo la ausencia de afecciones o enfermedades, para aceptar su concepto amplio de verdadero estado de bienestar físico y mental del trabajador.

Con base en estas definiciones, tendencias actuales apuntan a la modelación de todos los sistemas, y en especial el de seguridad y salud ocupacional, dentro de la planificación estratégica de la empresa, con el fin de vincular los objetivos de producción a los de prevención y vincular de forma directa las tareas de seguridad como puntos intrínsecos a las actividades de cada área, enmarcados con su objetivos estratégicos (Ulloa-Enríquez, 2012). [36-37]

En el Ecuador, mediante el programa de seguridad y salud en el trabajo, concurren a los siguientes objetivos para todo sistema de gestión en el área:

- Mejorar las condiciones de los trabajadores referentes a seguridad y salud. 
- Desarrollar consciencia preventiva y hábitos de trabajo seguros en empleadores y trabajadores.

- Disminuir las lesiones y daños a la salud provocados por el trabajo.

- Mejorar la productividad en base a la gestión empresarial con visión preventiva (Ministerio de Trabajo del Ecuador, 2014).

\section{Principales Sistemas de Gestión de Seguridad y Salud}

Un Sistema de Gestión de la Seguridad y Salud en el trabajo (SGSST) tiene como principio fomentar el entorno de trabajo seguro y saludable para sus colaboradores, ofreciendo un marco que permite, a los responsables, identificar y controlar los riesgos encontrados y nacientes de las actividades propias de la organización, reducir el potencial de accidentabilidad, cumplir con la normativa legal vigente en el país y mejorar el rendimiento y eficiencia laboral (Organización Internacional del Trabajo, 2011).

Las características principales de los Sistemas se basan en su accionar según el modelo de Deming: planificar, hacer, verificar y actuar (PHVA), lo que permite a la empresa una mejora integral de la competitividad, del servicio y del producto, mejorando la eficiencia y rentabilidad de la organización (Chávez Orozco C, 2013). En la figura 1. se muestra los pasos del ciclo PHVA para el desarrollo de la mejora continua en las empresas. 


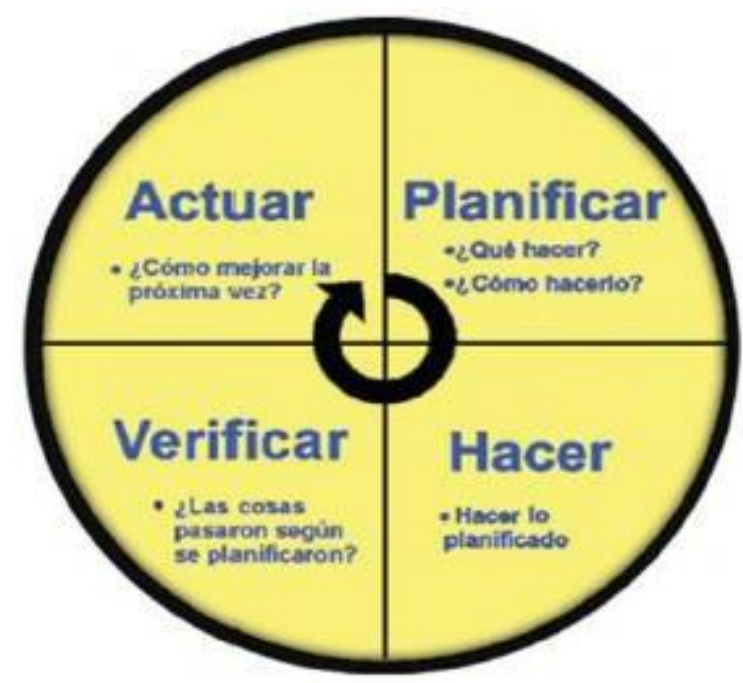

Figura 1. Ciclo de Deming

Son varias las instituciones y organismos internacionales que ofrecen sus metodologías para la aplicación de un sistema de gestión que garantice la seguridad y salud ocupacional, la tabla 1.2 muestra todas estas metodologías a nivel mundial. 
Dom. Cien., ISSN: 2477-8818

Vol. 3, núm. 4, octubre, 2017, pp. 264-283

Implantación de un sistema de gestión de seguridad y Salud en el trabajo basado en el modelo Ecuador

\begin{tabular}{|c|c|c|c|c|}
\hline & Palc & Recponcable & Reterenols & $\begin{array}{l}\text { Titulo del modelo, } \\
\text { borrador o norma }\end{array}$ \\
\hline 1 & $\begin{array}{l}\text { Rustralla y Nueva } \\
\text { Reianda }\end{array}$ & $\begin{array}{l}\text { gtandard Australla } \\
\text { gtandard New Zeland }\end{array}$ & $\begin{array}{l}\text { A3/ NZ3 } \\
4804: 1997\end{array}$ & $\begin{array}{l}\text { Occupational health and } \\
\text { safety manspement gystems } \\
\text { General guldellnes on } \\
\text { grinciples, system s and } \\
\text { supporting technlques }\end{array}$ \\
\hline 2 & $\begin{array}{l}\text { Nuatralis, region de } \\
\text { Vetoris }\end{array}$ & $\begin{array}{l}\text { Health and } 3 \text { afety } \\
\text { Prgarization }(\mathrm{HQO}), \\
\text { Metoris }\end{array}$ & Bafety Map & $\begin{array}{l}\text { Bafety Mansgement } \\
\text { Achievement Program ( } \\
\text { Bafety Map) }\end{array}$ \\
\hline 3 & Unien Europes & $\begin{array}{l}\text { Comise consultivo de la } \\
\text { Union Europes }\end{array}$ & Doc. 0135/4/99 EN & $\begin{array}{l}\text { European guidelines on the } \\
\text { zuccesstul organization of } \\
\text { zafety and health protection } \\
\text { for works at work (Batety } \\
\text { and heaith mansgement } \\
\text { zystema) }\end{array}$ \\
\hline 4 & inda & Ministerio de Trabajo & $\begin{array}{l}\text { Beccion } 41 F \text { del } \\
\text { Decrebo de } \\
\text { Fabricas de } 1948 \text {, } \\
\text { reviasdo en } 1988\end{array}$ & Diversos \\
\hline 6 & Intemacional & $\begin{array}{l}\text { Qil Induetry Intemational } \\
\text { Exploration and } \\
\text { Froduction Forum (ESP } \\
\text { Forum) }\end{array}$ & & $\begin{array}{l}\text { Guldelines for the } \\
\text { Development and } \\
\text { Application } \\
\text { of Heath, Safety and } \\
\text { Environmenty } \\
\text { Mansgement Systems }\end{array}$ \\
\hline 8 & Intemacional & $\begin{array}{l}\text { oo / Comlte Tecrico } 67 \text {, } \\
\text { Gubcombe } 6 \\
\text { Grupo de Trabajo } 1\end{array}$ & $\begin{array}{l}\text { Borwd } 14690 \\
\text { N46 rev. } 2\end{array}$ & $\begin{array}{l}\text { Petroleum and nature gas } \\
\text { ndustries- Hea'th, Safety } \\
\text { and Environmental } \\
\text { Management Systems. }\end{array}$ \\
\hline 7 & Intemacional & DHВАЗ & $\begin{array}{l}\text { OHBAB } \\
18001: 1999\end{array}$ & $\begin{array}{l}\text { Occupationa' healh and } \\
\text { Bafety Manspement } \\
\text { Bystems opecrication. }\end{array}$ \\
\hline 8 & Internacional & $\begin{array}{l}\text { Froyecto de Directrices de } \\
\text { a OIT }\end{array}$ & $\begin{array}{l}\text { LOVOOH-MB } \\
2001\end{array}$ & $\begin{array}{l}\text { Proyecto de drectrices } \\
\text { fecnicas la OIT sobre } \\
\text { alstemas de gestion de la } \\
\text { seguridad y aslud en } \\
\text { el trabajo. }\end{array}$ \\
\hline 8 & Intemacional & $\begin{array}{l}\text { PuPont de Nemourz. } \\
\text { Batety and Environment } \\
\text { Mansoement Services }\end{array}$ & DuFont & $\begin{array}{l}\text { Modeio de la gestion de la } \\
\text { zeguridad y de medlo } \\
\text { amblente de DuPont. }\end{array}$ \\
\hline 10 & rianda & $\begin{array}{l}\text { National Standards } \\
\text { Numority of Ireland }\end{array}$ & $\mathrm{OH}$ and $\mathrm{B}$ & $\begin{array}{l}\text { Drat Standard for Code of } \\
\text { Practice for an occupational } \\
\text { Heath Safety (OH and B) } \\
\text { Management Syatem 11. }\end{array}$ \\
\hline
\end{tabular}


Dom. Cien., ISSN: 2477-8818

Vol. 3, núm. 4, octubre, 2017, pp. 264-283

Implantación de un sistema de gestión de seguridad y Salud en el trabajo basado en el modelo Ecuador

\begin{tabular}{|c|c|c|c|c|}
\hline \multicolumn{2}{|r|}{ Palic } & Recponcable & Fibleronoly & $\begin{array}{l}\text { Thtula del modelo, } \\
\text { bort dor o noma }\end{array}$ \\
\hline 11 & famalca & $\begin{array}{l}\text { famalca Euread or } \\
\text { fondsrd }\end{array}$ & $\begin{array}{l}\text { Eomador oHas } \\
1-2\end{array}$ & 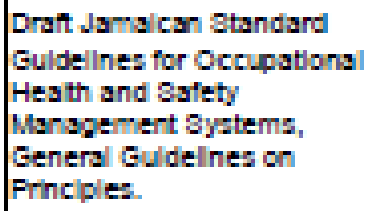 \\
\hline 12 & repdn & $\begin{array}{l}\text { Hapan Industr|ai Bstety s } \\
\text { Heath Acecclation }\end{array}$ & | Harro de 1997 & 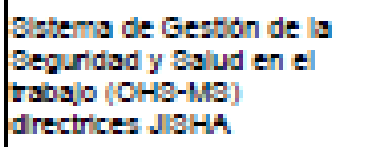 \\
\hline 13 & Porea & $\begin{array}{l}\text { Mirlsterlo de Trabajo, } \\
\text { Fepublica de Cores. }\end{array}$ & 1980 & 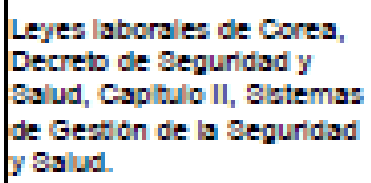 \\
\hline 14 & Holanda & $\begin{array}{l}\text { Nederiands Nomal abe } \\
\text { Inettute NFF sodi }\end{array}$ & & $\begin{array}{l}\text { lame ttcrico holandte } \\
\text { Gula para el Bstems de } \\
\text { Gestibn de la Bepuridad y } \\
\text { Galud en el trabojo. }\end{array}$ \\
\hline 16 & Honepa & Fonpes Standard|- & $\begin{array}{l}27 \text { de Aposto de } \\
1965\end{array}$ & 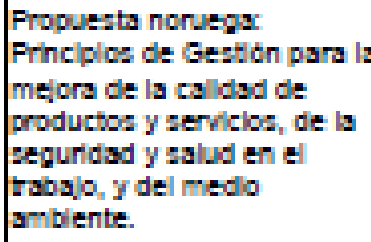 \\
\hline 16 & Folonla & $\begin{array}{l}\text { Frograma PHAFE de la } \\
\text { Inapection Laboral del } \\
\text { Etado Polsco }\end{array}$ & $\begin{array}{l}\text { Froprams de } \\
\text { Protecelon del } \\
\text { prabsjador PL } 9407 \\
\text { Nowlembre } 1996\end{array}$ & 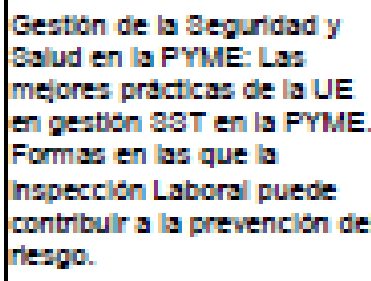 \\
\hline 18 & Pudatica & $\begin{array}{l}\text { Aatonal Occupational } \\
\text { fádety Avecolaton }\end{array}$ & 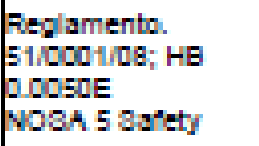 & Hes th Manspement Gystem \\
\hline 17 & Espaifa & $\begin{array}{l}\text { Hooclacion Epotola de } \\
\text { Homal|zadion } y \\
\text { Pertificadion }\end{array}$ & $\begin{array}{l}\text { UNE S1SOO } \\
\text { Diclembre 19SS }\end{array}$ & 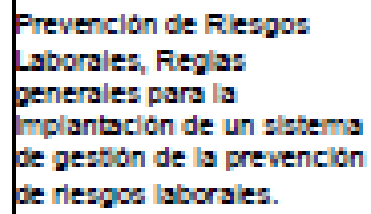 \\
\hline
\end{tabular}


Dom. Cien., ISSN: 2477-8818

Vol. 3, núm. 4, octubre, 2017, pp. 264-283

Implantación de un sistema de gestión de seguridad y Salud en el trabajo basado en el modelo Ecuador

\begin{tabular}{|c|c|c|c|c|}
\hline \multicolumn{2}{|r|}{ Palc } & Recponcable & Roforonola & $\begin{array}{l}\text { Thtulo dal modalo, } \\
\text { bomador o noma }\end{array}$ \\
\hline 18 & Espafia & Howoter consultores & THäU & $\begin{array}{l}\text { Total Hesth s botety } \\
\text { Manspemenit }\end{array}$ \\
\hline 19 & Feing Lnido & Ertah atandards & E5 9801: 1955 & 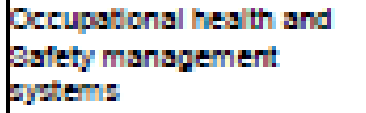 \\
\hline 20 & EEUU & $\begin{array}{l}\text { Amerlcan Industrial } \\
\text { Hglene Aacelselon }\end{array}$ & $\begin{array}{l}\text { AHAA OHEMG } \\
\text { 992R6 }\end{array}$ & $\begin{array}{l}\text { Sccupatonal Hesth and } \\
\text { Esiety Manapement, } \\
\text { Gytem: Mr AHA } \\
\text { Guldance Document }\end{array}$ \\
\hline 21 & EEUU & 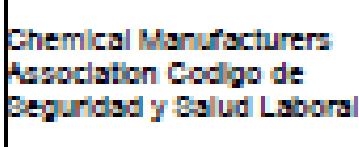 & Aesponsable care: & $\begin{array}{l}\text { Resoure Gulde tor the } \\
\text { Employes Heath and } \\
\text { Culety Code of } \\
\text { Manspement Fractlos }\end{array}$ \\
\hline 22 & EEUU & $\begin{array}{l}\text { Occuptonsi Bafety and } \\
\text { Hearh AdmInLstratbon }\end{array}$ & $\begin{array}{l}\text { Fedenal Repleter } \\
\text { 411208 }\end{array}$ & $\begin{array}{l}\text { Woluntary Protecton } \\
\text { Programs }\end{array}$ \\
\hline 23 & EEIUU & 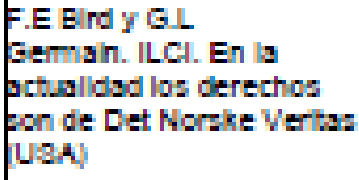 & $\begin{array}{l}\text { Control Total de } \\
\text { Ftrd ds: }\end{array}$ & Control Tosi de Ferdids. \\
\hline 24 & EEIUU & $\begin{array}{l}\text { Decupabonsl Bafety and } \\
\text { Hearh Adminlatration }\end{array}$ & $1,910,700$ & $\begin{array}{l}\text { Prat Propese Es'ety an } \\
\text { Healt Frogram Etsidard. }\end{array}$ \\
\hline 26 & EEIUU & 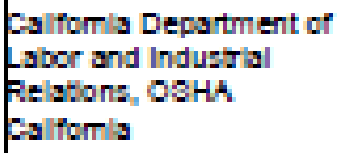 & $\begin{array}{l}\text { Tomo } 12 \text {, apotado } \\
\text { E, parte } 2 \text { capitulo } \\
\text { oo-2 }\end{array}$ & $\begin{array}{l}\text { Beneral Bafety and Health } \\
\text { Fequirement: Bafety and } \\
\text { Healt Froprams }\end{array}$ \\
\hline 29 & Ecuador & Eos & $\begin{array}{l}\text { Decrebo 2393, } \\
\text { BART. }\end{array}$ & Modelo Ecusdar \\
\hline
\end{tabular}

Directrices ILO-OSH 2001

Según (Organización Internacional del Trabajo, 2001), las Directrices ILO-OSH 2001 "ofrecen un modelo internacional excepcional, compatible con otras normas y pautas sobre el sistema de gestión”. Dichas directrices sobre seguridad y salud en el trabajo son de uso voluntario, poseen un enfoquetripartito, proporciona fortaleza, flexibilidad y bases adecuadas para la fomentación de una cultura enfocada a la protección de los trabajadores. Este tipo de 
recomendaciones sirven de complemento a las leyes y reglamentos nacionales, y no exige certificación ni aprobación internacional.

La figura 1.5 muestra un resumen de las directrices del modelo para su implantación dentro de una organización empresarial, con el objetivo final de establecer la mejora continua como resultado de su proceso productivo.

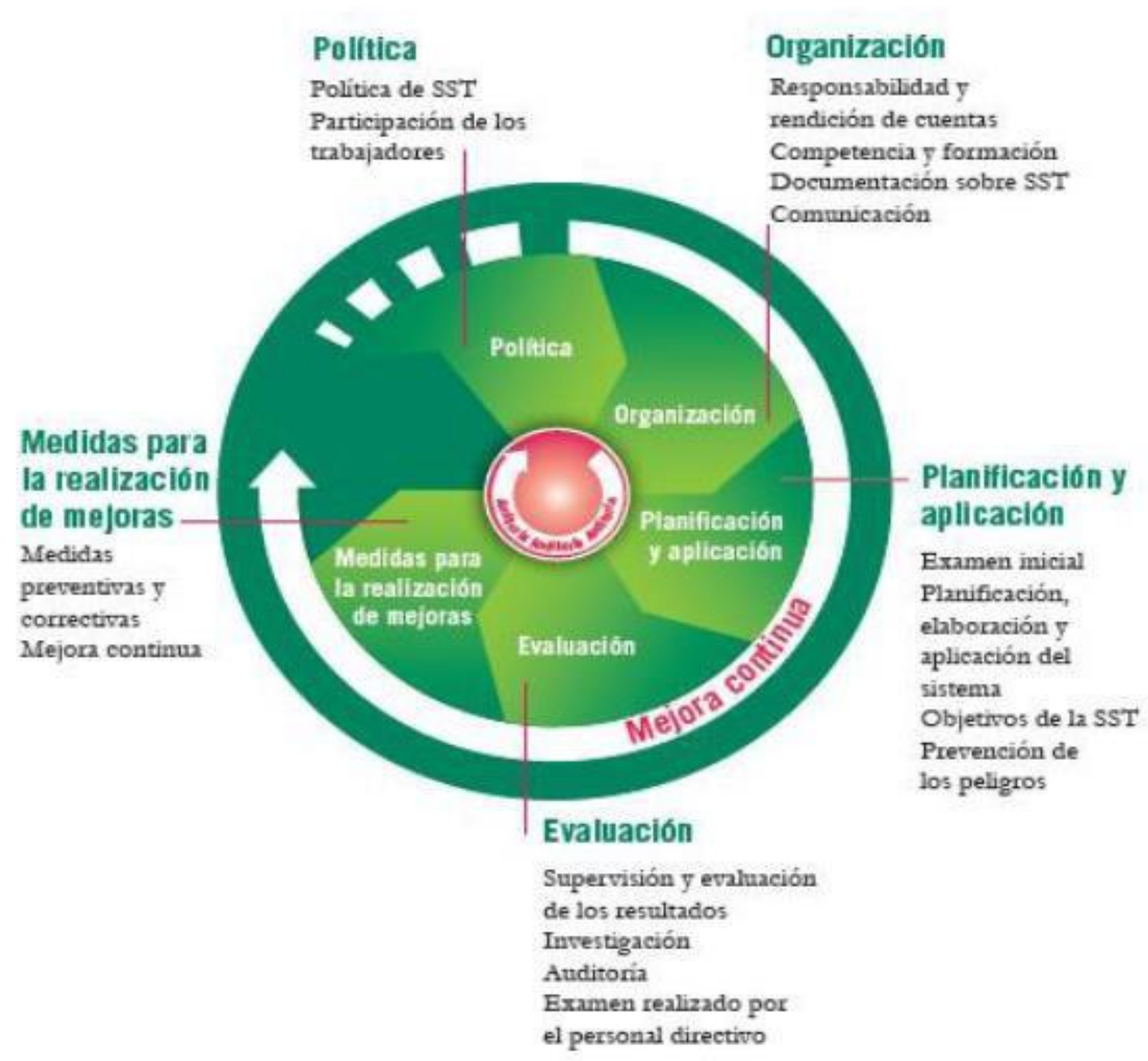

Figura 1.5: Directrices de la OIT relativas a los SG-SST: el ciclo de mejora continua 
El Sistema de Seguridad y Salud OHSAS (por su terminología en inglés Occupational Health and Safety Assessment Series) materializadas por British Standards Institution (BSI), proporciona los elementos de un sistema que permite ser integrado con otros sistemas, especialmente los relativos a sus familias ISO 9001 e ISO 14001.

Los principios de la normativa voluntaria internacional, se relacionan con los estimados en la Directrices ILO-OSH 2001, partiendo del establecimiento de una política y unos objetivos según los requisitos legales nacionales. Este sistema hace énfasis en la prácticas proactivas preventivas de la identificación de peligros o factores de riesgos y la evaluación y control de los mimos de forma inmediata. (Chávez Orozco C, 2010). El éxito del sistema depende del compromiso de todos los niveles y funciones de la organización, y especialmente de los altos mandos.

La figura 1.6 muestra los pasos de implantación de un sistema de gestión según la normativa OHSAS 18001, con el objetivo final, como todo sistema de gestión, de establecer la mejora continua. 


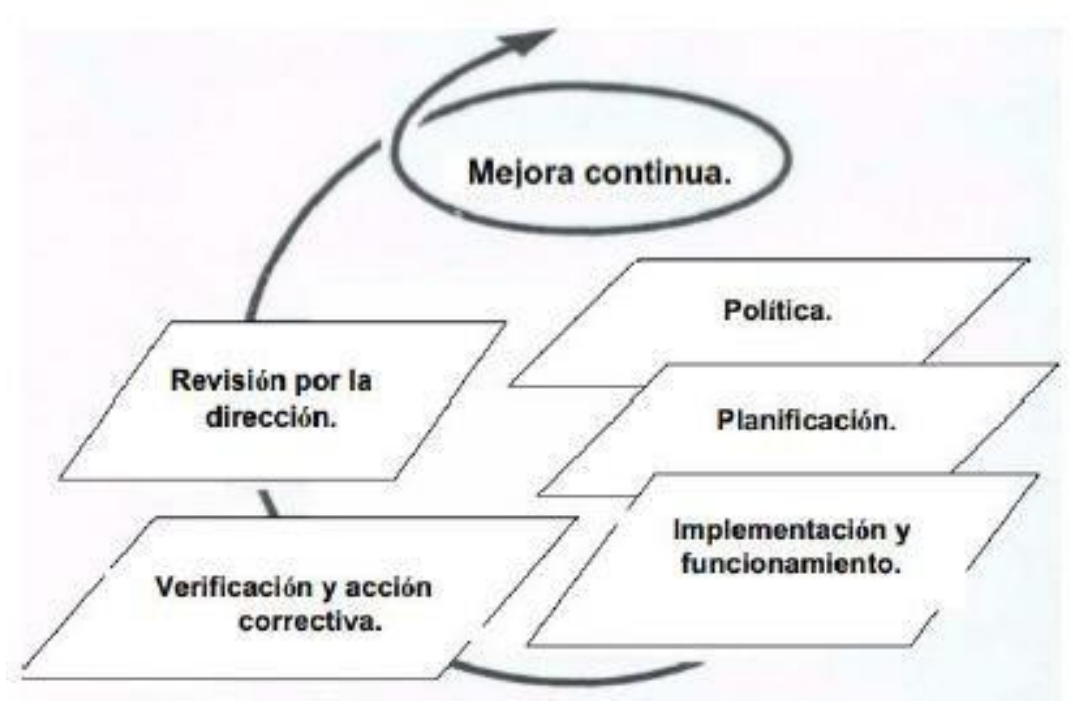

Figura 1.6: Modelo del Sistema de Gestión OHSAS

Sistema de Gestión de Seguridad y Salud en el Trabajo "MODELO ECUADOR”

El Modelo Ecuador, es un modelo de gestión establecido por la Dirección del Seguro General de Riesgos del Trabajo del IESS, el mismo que centra su objetivo en la prevención de riesgos laborales.

Según (Vásquez Zamora, 2011) el modelo sirvió como base al Reglamento del Instrumento Andino de Seguridad y Salud en el Trabajo, Resolución 957, que es la vinculante para los cuatros países de la subregión andina, y que en su artículo primero recomienda la aplicación del sistema de gestión que comprende los elementos y subelementos que tiene el Modelo Ecuador.

En octubre de 2010 el Seguro General de Riesgos del Trabajo del IESS publicó el Sistema de Auditorias de Riesgos del Trabajo (SART) mediante la Resolución 333, dándole aplicabilidad efectiva al mismo. 
En la figura 1.7 muestra los elementos del Sistema Gestión de Seguridad y Salud en el Trabajo según el Modelo Ecuador.

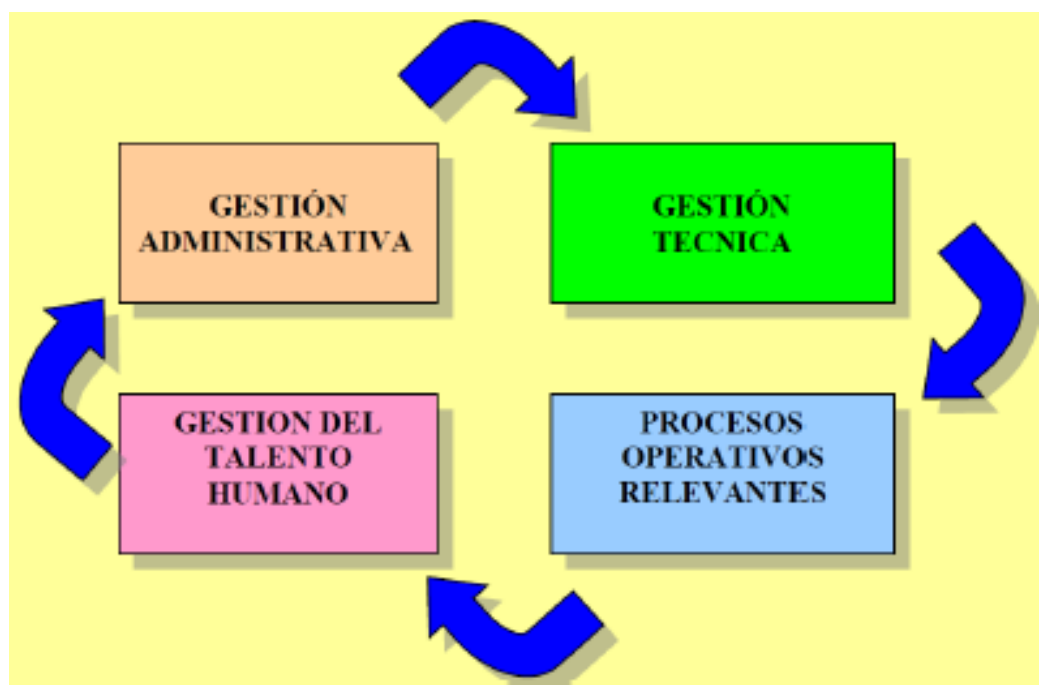

Figura 1.7: Elementos del Modelo Ecuador

Cada uno de estos elementos representa una esfera de actuación dentro de las organizaciones, cuya interrelación eficaz permitirá el cumplimiento de un sistema que garantiza la prevención de riesgos laborales.

\section{Conclusiones.}

1. La Unidad Educativa “Isaac Newton” de la ciudad de Manta, carecía de un sistema de gestión en seguridad y salud en el trabajo por lo que en la auditoría de diagnóstico de la situación inicial a través de la plataforma del Sistema Nacional de Gestión de la Prevención bajo la metodología del SART, obtuvo un índice de eficiencia del 6,56\% de cumplimiento. 
2. El índice de eficiencia alcanzado en la evaluación inicial es considerado como insatisfactorio por la normativa legal correspondiente por lo que acarrea al cumplimiento de multas pecuniarias y administrativas de los organismos de control.

3. Considerando la auditoria inicial, se planificó y temporizó las actividades a desarrollar para la implantación del sistema de gestión: en al ámbito administrativo se estableció y difundió la política integrada de seguridad, se conformó sus unidades de prevención: comité paritario, departamento de seguridad (integrado por el técnicode seguridad, médico ocupacional y psicólogo), se estableció la planificación del sistema y se propuso el Reglamento Interno de Seguridad e Higiene Laboral de la institución para su registro en el Ministerio de Relaciones Laborales de la localidad. Dentro de la gestión técnica se identificó los factores de riesgos expuestos en todos los puestos de trabajo de la institución, demostrando que el 32,54\% corresponde a mecánicos, el 27,89\% son psicosociales, el 21,62\% son ergonómicos, el 12,88\% son físicos, el 4,68\% son químicos y el 0,38\% son biológicos; centrándose las medidas correctivas en los actividades del departamento de mantenimiento y en área académica de la institución. Bajo la metodología descrita en el talento humano del Modelo Ecuador, se estableció los procesos de selección de personal considerando los riesgos expuesto mediante la elaboración de los profesiogramas por puesto de trabajo, programa de inducción y establecimiento de procesos de comunicación e información del sistema. Dentro de los procesos operativos básicos, se establecieron los controles médicos, desarrollándose exámenes periódicos a todo el personal, se distribuyó los EPP a los puestos requeridos según profesiogramas, y se identificó y corrigió condiciones subestándares de trabajo. Para la implantación y control del sistema se crearon dieciséis procedimientos y treinta formatos. 
4. Con los programas implantados y actividades desarrolladas según la planificación del sistema de gestión, en una nueva auditoría se logró alcanzar un índice de cumplimiento del 68,73\% en la gestión administrativa, 47,67\% en la gestión técnica, 79,33\% en la gestión de talento humano y un $73,06 \%$ en los procedimientos y programas operativos básicos, alcanzado un índice de eficiencia global del 68,02\%. De igual forma, se dejaron planteados propuestas de implantación en un 23,37\% para el cumplimiento de los requisitos técnicos legales de la metodología, alcanzando a futuro un índice de eficiencia del $81,39 \%$ considerado como satisfactorio.

5. La evaluación económica del proyecto resultó favorable dado que en sus análisis: el VAN, con un 8\% de tasa mínima de retorno, resultó en $\$ 14748,17$ por encima de los egresos, considerado beneficioso; la relación beneficio/costo resultó en un valor de 1,17 por lo que por cada dólar que la institución invierta en el sistema de gestión de seguridad y salud en el trabajo se tiene una ganancia de 0,17 dólares; y su tasa interna de retorno se entabló en $81,38 \%$ considerado factible y ejecutable.

\section{Bibliografía.}

1) Arias Orellana, A., Arias Orellana, A., \& Pisco Merchán, M. (2012). Diseño de un sistema de seguridad y salud ocupacional para una empresa que se dedica a la importación y comercialización de respuestas electrónicos alineados a SART. Guayaquil: Escuela Superior Politécnica del Litoral.

2) Armas Ruiz, G. (2009). Diseño de un Sistema de Gestión de Prevención de Riesgos Laborales para el empresa florícola Jardines Piaveri Cía. Ltda. Basada en el Modelo Ecuador. Quito: Universidad San Francisco de Quito y Universidad de Huelva -España.

3) Asamblea Nacional del Ecuador. (1986). (Decreto Ejecutivo 2393) Reglamento de Seguridad y Salud de los Trabajadores y Mejoramiento del Medio Ambiente del Trabajo. Quito: Registro Oficial N 565.17 noviembre de 1986. Última modificación 21 febrero 2003.

4) Asamblea Nacional del Ecuador. (2008). Constitución del Ecuador. Quito: Registro Oficial $\mathrm{N}^{\circ} 449.20$ octubre 2008.

5) Asamblea Nacional del Ecuador. (2011). Código del Trabajo. Quito: Registro Oficial Suplemento 167. 16 diciembre 2005 Última modificación: 27 enero 2011.

6) Asamblea Nacional del Ecuador. (2014). Ley de seguridad social. Quito: Registro Oficial Suplemento 465 de 30-nov-2001. Última modificación: 10-feb-2014.

7) Azcuénaga Linaza, L. (2006). Manual Práctico para la Investigación de accidentes e incidentes laborales. 2da edición. Madrid: Fundación Confemetal. ISBN 84-96169-82-0. 
8) Beltrán Sanz, J., Carmona Calvo, M., Carrasco Perez, R., Rivas Zapata, M., \& Tejedor Panchon, F. (2014). Guía para una gestión basada en procesos. Bilbao, España: Instituto Andaluz de Tecnología. ISBN 84-923464-7-7.

9) Benemérito Cuerpo de Bomberos de Manta. (2014). Instructuvo para el desarrollo del Plan de Emergencia para las Unidades Educativas. Manta: Editorial no especificado.

10) Cañada Clé, J., Diáz Olivares, I., Medina Chamarro, J., Puebla Hernánz, M., Simón Mata, J., \& Soriano Serrano, M. (2009). Manual para el profesor de Seguridad y Salud en el Trabajo. Barcelona: Instituto Nacional de Seguridad e Higiene en el Trabajo. ISBN 978-84-7425-7632.

11) Chávez Orozco, C. (2010). Enfoque Estratégico de la Seguridad y Salud Ocupacional. EIDOS, 22-30. ISSN: 1390-5007.

12) Chávez Orozco, C. (2013). Gestión de la Seguridad y Salud en el Trabajo. Eídos 2, 14-18. ISSN 1390-499X.

13) Comunidad Andina. (2005). (Decisión 584). Instrumento Andino de Seguridad y Salud en el Trabajo. Consejo Consultivo Laboral Andino. Lima: Instituto Laboral Andino. Primera Edición. Depósito Legal: 20057599.

14) Comunidad Andina. (2005). (Resolución 957) Reglamento del Instrumento Andino de Seguridad y Salud en el Trabajo. Lima. : Instituto Laboral Andino. Depósito Legal: 20057599.

15) Espinoza de los Monteros Fernández C. (2010). Modelo de Gestión en Seguridad y Salud Modelo Ecuador, Diseño de Gestión en la Empresa Entrix América S.A. Departamento de Salud Comunitaria. Quito: Universidad San Francisco de Quito y Universidad de HuelvaEspaña.

16) Fernández, F., Iglesias Pastrana, D., Llaneza Álvarez, F., \& Fernández Muniz, B. (2010). Manual para la formación del auditor en prevención de riesgos laborales. Aplicaciones y casos prácticos. Tercera Edición. Valladolid, Valladolid, España: Lex Nova. ISBN 978-849898-155-1.

17) Granados P., I. (2011). Calidad de vida laboral, historia dimensiones y beneficios. Quality of working life: history, dimensions and benefits. IIPSI, 271-276. ISSN 1609 - 7445.

18) Instituto Ecuatoriano de Seguridad Social. (2010). (Resolución $N^{\circ}$ C.D. 333) Reglamento para el Sistema de Auditorías de Riesgo del Trabajo. Quito: Registro Oficial $\mathrm{N}^{\circ} 319.12$ de noviembre de 2010.

19) Instituto Ecuatoriano de Seguridad Social. (2011). (Resolución No. C.D.390) Reglamento del Seguro General del Riesgo del Trabajo. Consejo Directivo. Quito: Registro Oficial $\mathrm{N}^{\circ} 599$. 19 de diciembre del 2011.

20) Instituto Ecuatoriano de Seguridad Social. (2012). Seguro del Riesgos del Trabajo. Recuperado el 10 de 04 de 2015, de Mandatos legales en seguridad y salud acorde al tamaño de la empresa: http://www.higieneindustrialyambiente.com/userfiles/obligacionesempresariales-tamanio-de-empresa.pdf

21) Leones Vásquez, P. (2011). Plan de prevención de riesgos laborales en la empresa Randimpak de la ciudad de Riobamba. Riobamba: Escuela Superior Politécnica del Chimborazo.

22) Luna Rosauro, F. (2011). Prevención de Riesgos Laborales. Málaga: Vértice S.L. ISBN 97884-9931-514-0. 
23) Ministerio de Relaciones Laborales - Instituto Ecuatoriano de Seguridad Social. (2014). Instructivo para la implementación del Sistema Nacional de Gestión de Prevención de Riesgos Laborales (SGP). Quito: Registro Oficial N¹96 - 6 marzo 2014.

24) Ministerio de Relaciones Laborales. (2012). El manual de requisitos y definición del trámite de aprobación del reglamento de seguridad y salud. . Quito: Registro Oficial Segundo Suplemento $\mathrm{N}^{\circ}$ 845. 05 diciembre de 2012.

25) Ministerio de Trabajo. (5 de 06 de 2014). Recuperado el 10 de 04 de 2015, de Auditorías Del SGP-Prevención de riesgos laborales inician el 06 de junio: http://www.trabajo.gob.ec/auditorias-del-sgp-prevencion-de-riesgos-laborales-inician-el-06de-junio/

26) Ministerio de Trabajo del Ecuador. (2014). Sistema de Gestión de Seguridad y Salud del Ministerio de Relaciones Laborales. Recuperado el 15 de 01 de 2015, de http://www.trabajo.gob.ec/seguridad-y-salud-en-el-trabajo/

27) Ministerio de Trabajo y Bienestar Social (Actual Ministerio del Trabajo del Ecuador). (1981). (Acuerdo $N^{\circ}$ 1404) Reglamento para el funcionamiento de los servicios médicos de empresas. Quito: Registro Oficial 698.

28) OHSAS Project Group. (2007). OHSAS 18001-2007 Occupational health and safety management systems - requirements. Madrid. ISBN 978058050802 8: AENOR.

29) Organización Internacional del Trabajo. (2001). Directrices relativas a los sistemas de gestión de la seguridad y la salud en el trabajo ILO-OSH. Oficina Internacional del Trabajo, Ginebra. ISBN 92-2-311634-1.

30) Organización Internacional del Trabajo. (2011). Sistema de Gestión de la SST: una herramienta para la mejora continua. lera edición. Turín, Italia: Centro Internacional de Formación de la OIT. ISBN 978-92-2-324740-9.

31) Real Academia Española. (2014). Diccionario de la Lengua Española. Madrid, España: RAE, $23 e r a$ Edición. ISBN-13: 978-8467041897.

32) Secretaría Nacional de Planificación y Desarrollo. (2013). Plan Nacional de Desarrollo / Plan Nacional para el Buen Vivir 2013-2017 (Primera ed.). Quito, Ecuador: Senplades ISBN-978-9942-07-448-5.

33) Ulloa-Enríquez, M. (2012). Riesgos del Trabajo en el Sistema de Gestión de Calidad. Risks of the Work in the Quality Management System. Ingeniería Industrial, 24-31. ISSN 18155936.

34) Unidad Educativa Particular Isaac Newton. (2014). Plan de Emergencia Institucional. Manta: UEIN.

35) Unidad Educativa Particular Isaac Newton. (2014). Plan Operativo Anual. Manta: UEIN.

36) Vásquez Zamora, L. (2011). Gestión Integral e Integrada de Seguridad y Salud Modelo Ecuador. (I. E. Social, Ed.) Recuperado el 10 de 04 de 2015, de Revista Técnica Informativa del Seguro General de Riesgos del Trabajo. Ecuador: http://www.iess.gob.ec/documents/10162/51889/Revista-edicion1.pdf

37) Vásquez Zamora, L. (2014). Auditorías del Riesgo del Trabajo II. 14-16. Manta, Manabí, Ecuador: Universidad San Francisco de Quito. 